\title{
Fucoxanthin, A Xanthophyll from Macro- and Microalgae: Extraction Techniques, Bioactivities and Their Potential Application in Nutra- and Cosmeceutical Industries
}

\author{
Dedi Noviendri ${ }^{1, *}$, Diini Fithriani ${ }^{1}$, and Reno F. Hasrini ${ }^{2}$ \\ ${ }^{1}$ Research Center for Marine and Fisheries Product Processing and Biotechnology. Ministry of \\ Marine Affairs and Fisheries. Republic of Indonesia. Jl. KS Tubun Petamburan VI. Central Jakarta. \\ Indonesia. \\ ${ }^{2}$ Center of Agro-Based Industry (BBIA), Ministry of Industry, Republic Indonesia. J1. H. \\ Juanda No 11 Bogor, West Java, Indonesia
}

\begin{abstract}
Some works of literature reported that fucoxanthin has diverse potential benefits for human health. Thus, this review would explain the sources of fucoxanthin, extraction techniques, bioactivities, and its potential application in Nutra- and cosmeceutical industries. Brown algae, such as Padina australis, Undaria pinnatifida; and the microalgae, such as Chaetoceros gracilis, Phaeodactylum tricornutum were sources of fucoxanthin. Then, the chemical structure of this xanthophyll is unique and that confers its biological activities. And then, the extraction process of fucoxanthin from macro- and microalgae is more safe, accessible, and economic, although this xanthophyll can be synthesized chemically. Generally, there are two techniques for the extraction of fucoxanthin, namely liquid solvent (conventional), and supercritical carbon dioxide (non-conventional) extractions. Furthermore, there are some bioactivities of fucoxanthin, including its activities of anticancer, antidiabetic, antiobesity, antioxidant; protective effects of skin, bone, and eyes. Based on in vivo assay of the animal, it has no adverse effects of fucoxanthin supplementation. Therefore, this xanthophyll might be applied in both the Nutra- and cosmeceutical industries. In the future, fucoxanthin and its derivatives would be important for human health, contributing to the beauty industry, and playing an important in the prevention of cancer and the disease related to lifestyle.
\end{abstract}

\section{Introduction}

About 800 kinds of xanthophylls have been reported in nature up until 2018 [1]. This class of carotenoid, especially fucoxanthin is abundant in macro- and microalgae as a photoprotection component, and the complex of light-harvesting for the photosynthesis process [2]. The fucoxanthin chemical structure contains a 5.6-monoepoxide and an allenic

\footnotetext{
*Corresponding author: dedinov@yahoo.com
} 
bond [3] (Fig. 1). Its chemical structure is unique and that confers its biological activities. And then, some kinds of literature reported that fucoxanthin has diverse potential benefits for human health [4]. So, this review would explain the sources of fucoxanthin, its extraction techniques, bioactivities, and its potential application in both the Nutra- and cosmeceutical industries.

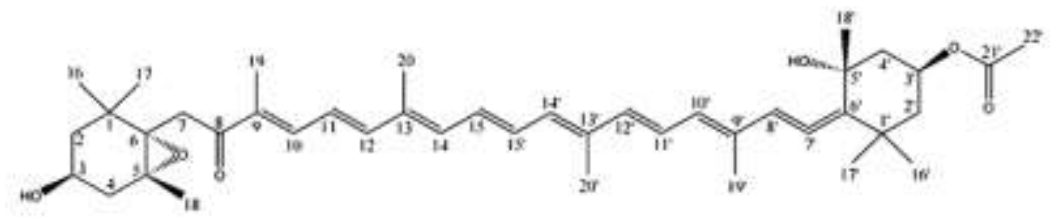

Fig. 1. The fucoxanthin chemical structure

\section{Sources of Fucoxanthin}

Fucoxanthin is found in macroalgae, especially brown algae, and microalgae, or a number of diatoms [5-7]. This compound is a major xanthophyll carotenoid in the chloroplast of brown algae [8], namely Padina australis [9], Sargassum horneri [10], Undaria pinnativida [11], Laminaria japonica [12]; in microalgae or diatoms [13] such as Phaeodactylum tricornutum [14], Chaetoceros gracilis [15], Isochrysis galbana [16]; and a small amount in red seaweed, such as Chondria crassicaulis, Mazzaella japonica, and Gloiopeltis furcate [17]. The content of fucoxanthin in brown algae is about $0.1-1.0 \mathrm{mg} / \mathrm{g}(0.01-0.10 \%)$ of the dry cell weight [18], while in diatoms is about $1.00-2.50 \%$ of the dry cell weight $[19,20]$. The sources data of fucoxanthin from macro and microalga (from years 2018 to 2020) are shown in Table 1.

Table 1. The sources of fucoxanthin from macro and macroalga (from 2018 to 2020).

\begin{tabular}{|c|c|c|c|c|}
\hline Year & Source & Species & Country & References \\
\hline \multirow[t]{24}{*}{2018} & \multirow[t]{24}{*}{ BS } & Alaria esculenta & Ireland & [21] \\
\hline & & Ascophyllum nodosum & Ireland & [21] \\
\hline & & Colpomenia sinuosa & Iran & [22] \\
\hline & & Dictyota indica & Iran & [22] \\
\hline & & Fucus serratus & Ireland & [21] \\
\hline & & Fucus vesiculosus & Ireland & [21] \\
\hline & & Himanthalia elongata & Ireland & {$[21]$} \\
\hline & & Iyengaria stellate & Iran & [22] \\
\hline & & Laminaria hyperborea & Ireland & [21] \\
\hline & & Laminaria digitata & Ireland & [21] \\
\hline & & Laminaria japonica & China & [23] \\
\hline & & Laminaria japonica & Korea & [12] \\
\hline & & Laminaria japonica & Taiwan & [24] \\
\hline & & Padina australis & Indonesia & [25] \\
\hline & & Padina tenuis & Iran & [22] \\
\hline & & Padina tetrastromatica & India & [26] \\
\hline & & Pelvetia canaliculata & Ireland & {$[21]$} \\
\hline & & Saccharina japonica & Korea & [27] \\
\hline & & Saccharina japonica & Korea & [29] \\
\hline & & Saccharina latissima & Ireland & [21] \\
\hline & & Saccorhiza polyschides & Ireland & [21] \\
\hline & & Sargassum polycystum & Malaysia & [29] \\
\hline & & Sargassum siliquosum & Malaysia & [29] \\
\hline & & Sphaerotrichia divaricata & Japan & [30] \\
\hline
\end{tabular}




\begin{tabular}{|c|c|c|c|c|}
\hline & & Turbinaria deccurrens & Indonesia & [31] \\
\hline & & Undaria pinnatifida & China & [32] \\
\hline & & Undaria pinnatifida & China & [33] \\
\hline & & Undaria pinnatifida & China & [34] \\
\hline & & Undaria pinnatifida & Japan & [35] \\
\hline & \multirow[t]{43}{*}{$\mathrm{MA} / \mathrm{D}$} & Chaetoceros calcitrans & Malaysia & [36] \\
\hline & & Cylindrotheca fusiformis & China & [37] \\
\hline & & Isochrysis galbana & Italy & [38] \\
\hline & & Nanofrustulum shiloi & Turkey & [39] \\
\hline & & Nitzschia $\mathrm{sp}$ & Turkey & [39] \\
\hline & & Nitzschia laevis & USA & [40] \\
\hline & & Nitzschia laevis & USA & [41] \\
\hline & & Odontella aurita & Denmark & [42] \\
\hline & & Phaeodactylum tricornutum & Australia & [43] \\
\hline & & Phaeodactylum tricornutum & China & [6] \\
\hline & & Phaeodactylum tricornutum & China & [44] \\
\hline & & Phaeodactylum tricornutum & Czech Republic & [45] \\
\hline & & Phaeodactylum tricornutum & Iceland & [46] \\
\hline & & Phaeodactylum tricornutum & Italy & [38] \\
\hline & & Phaeodactylum tricornutum & France & [47] \\
\hline & & Phaeodactylum tricornutum & Germany & [48] \\
\hline & & Phaeodactylum tricornutum & Korea & [49] \\
\hline & & Phaeodactylum tricornutum & Netherland & [50] \\
\hline & & Phaeodactylum tricornutum & Scotland & [46] \\
\hline & & Phaeodactylum tricornutum & USA & [37] \\
\hline & & Tisochrysis lutea & Italy & [51] \\
\hline \multirow[t]{28}{*}{2019} & & Achnanthidium sp. & Korea & [52] \\
\hline & & Amphora $\mathrm{sp}$ & Australia & [53] \\
\hline & & Conticribra weissflogii & China & [54] \\
\hline & & Dunaliela salina & India & [55] \\
\hline & & Isochrysis galbana & Chile & [56] \\
\hline & & Isochrysis galbana & India & [55] \\
\hline & & Isochrysis galbana & Portugal & [16] \\
\hline & & Navicula $\mathrm{sp}$ & India & [55] \\
\hline & & Nitzschia sp & China & [57] \\
\hline & & Nitzschia laevis & China & [58] \\
\hline & & Nitzschia laevis & China & [59] \\
\hline & & Nitzschia laevis & USA & [60] \\
\hline & & Pavlova lutheri & India & [55] \\
\hline & & Phaeodactylum tricornutum & China & {$[61,62]$} \\
\hline & & Phaeodactylum tricornutum & China & {$[63,65]$} \\
\hline & & Phaeodactylum tricornutum & Germany & [64] \\
\hline & & Phaeodactylum tricornutum & Germany & [65] \\
\hline & & Phaeodactylum tricornutum & Japan & [66] \\
\hline & & Phaeodactylum tricornutum & Korea & [67] \\
\hline & & Phaeodactylum tricornutum & Korea & [68] \\
\hline & & Skeletonema costatum & Indonesia & [69] \\
\hline & & Tisochrysis lutea & Iran & [70] \\
\hline & $\mathrm{BS}$ & Cystosphaera jacquinotii & Brazil & [71] \\
\hline & \multirow[t]{2}{*}{$\mathrm{RS}$} & Chondria crassicaulis & Japan & [72] \\
\hline & & Chondrus yendoi & Japan & [72] \\
\hline & \multirow[t]{3}{*}{ BS } & Costaria costata & Japan & [72] \\
\hline & & Desmarestia anceps & Brazil & [71] \\
\hline & & Eisenia bicyclis & Korea & [73] \\
\hline
\end{tabular}




\begin{tabular}{|c|c|c|c|c|}
\hline & $\mathrm{RS}$ & Gloiopeltis furcata & Japan & {$[72]$} \\
\hline & \multirow[t]{2}{*}{$\mathrm{BS}$} & Laminaria japonica & Taiwan & {$[74]$} \\
\hline & & Laminaria japonica & Japan & {$[75]$} \\
\hline & $\mathrm{RS}$ & Mazzaella japonica & Japan & {$[72]$} \\
\hline & \multirow[t]{11}{*}{$\mathrm{BS}$} & Saccharina japonica & Japan & {$[72]$} \\
\hline & & Saccharina japonica & Korea & [76] \\
\hline & & Sargassum aquifolium & Japan & {$[72]$} \\
\hline & & Sargassum fusiforme & Japan & {$[72]$} \\
\hline & & Sargassum horneri & Japan & {$[72]$} \\
\hline & & Undaria pinnatifida & Japan & {$[72]$} \\
\hline & & Undaria pinnatifida & Korea & {$[73]$} \\
\hline & & Undaria pinnatifida & Japan & {$[77]$} \\
\hline & & Undaria pinnatifida & China & {$[78]$} \\
\hline & & Undaria pinnatifida & China & {$[58]$} \\
\hline & & Undaria pinnatifida & Japan & {$[79]$} \\
\hline \multirow[t]{27}{*}{2020} & \multirow[t]{16}{*}{$\mathrm{MA} / \mathrm{D}$} & Chaetoceros calcitrans & Malaysia & {$[80]$} \\
\hline & & Chaetoceros gracilis & Japan & {$[81]$} \\
\hline & & Chaetoceros gracilis & Japan & {$[15]$} \\
\hline & & Chaetoceros gracilis & Japan & {$[14]$} \\
\hline & & Halamphora coffeaeformis & Argentina & {$[82]$} \\
\hline & & Nitzschia palea & Bergium & [83] \\
\hline & & Phaeodactylum tricornutum & China & {$[84]$} \\
\hline & & Phaeodactylum tricornutum & Japan & {$[85]$} \\
\hline & & Phaeodactylum tricornutum & Japan & [14] \\
\hline & & Phaeodactylum tricornutum & Korea & {$[86]$} \\
\hline & & Phaeodactylum tricornutum & USA & {$[87]$} \\
\hline & & Sellaphora minima & Belgium & {$[83]$} \\
\hline & & Thalassiosira weissflogii & India & {$[88]$} \\
\hline & & Tisochrysis lutea & France & [89] \\
\hline & & Tisochrysis lutea & Netherland & {$[90]$} \\
\hline & & Tisochrysis lutea & Portugal & [91] \\
\hline & \multirow[t]{11}{*}{$\mathrm{BS}$} & Desmarestia anceps & Brazil & {$[92]$} \\
\hline & & Himanthalia elongata & Spain & {$[11]$} \\
\hline & & Laminaria ochroleuca & Spain & {$[11]$} \\
\hline & & Padina australis & Indonesia & [9] \\
\hline & & Sargassum angustifolium & Iran & [93] \\
\hline & & Sargassum glaucescens & Canada & {$[94]$} \\
\hline & & Sargassum horneri & Japan & {$[95]$} \\
\hline & & Sargassum polycystum & Indonesia & {$[96]$} \\
\hline & & Sargassum wightii & India & [97] \\
\hline & & Undaria pinnatifida & Spain & {$[11]$} \\
\hline & & Alaria esculenta & Ireland & [21] \\
\hline
\end{tabular}

Note: BS: Brown seaweed; RS: Red Seaweed; MA/D: Microalga/Diatom

\section{Fucoxanthin Extraction}

Generally, there are two main techniques to extract fucoxanthin, namely liquid solvent extraction (conventional), and supercritical carbon dioxide $\left(\mathrm{SC}-\mathrm{CO}_{2}\right)$, usually use $\mathrm{CO}_{2}$ as a co-solvent (nonconventional) [98-100]. In the liquid solvent extraction with organic solvents, the recovery of solvent is a crucial period, mainly because of the environmental and economic problems. This technique has disadvantages, such as there are remind residue of solvent in the extract, the possible thermal degradation of the extract, and has low selectivity [101]. Additionally, the disadvantage of the liquid solvent extraction needed 
many solvents in its technique. However, the extraction technique with liquid organic solvents is the most effective in the extraction of carotenoids [102]. In contrast, the extraction with $\mathrm{SC}-\mathrm{CO}_{2}$ has an advantage, mainly due to the environment. This extraction technique is a process free of waste, prevents oxidation reactions, allow low-temperature use, does not cause thermal degradation of extract, and do not need the removal of solvent [103-105]. Additionally, this extraction technique has an advantage, mainly due to the economy, namely only needed a small amount of organic solvent, and needed a short time to the extraction process [106].

\section{Fucoxanthin and Their Biological Activities}

Fucoxanthin is a xanthophyll exhibiting several health benefits. This compound has remarkable biological properties for human health [109], such as anti-inflammatory activity [108-115], hepatoprotective effect [12,98,116-118], cardioprotective activity [119-121], anticancer [26,116,119,122-131], antidiabetic [75,132-134], antiobesity [135-139], antioxidant [65,131,140-146], skin protective effect [111,113,147-153], neuroprotective activity [75,155-161], osteoprotective effect $[162,163]$, and eyes protective effect [164166]. The biological activities of fucoxanthin for human health are shown in Fig. 2.

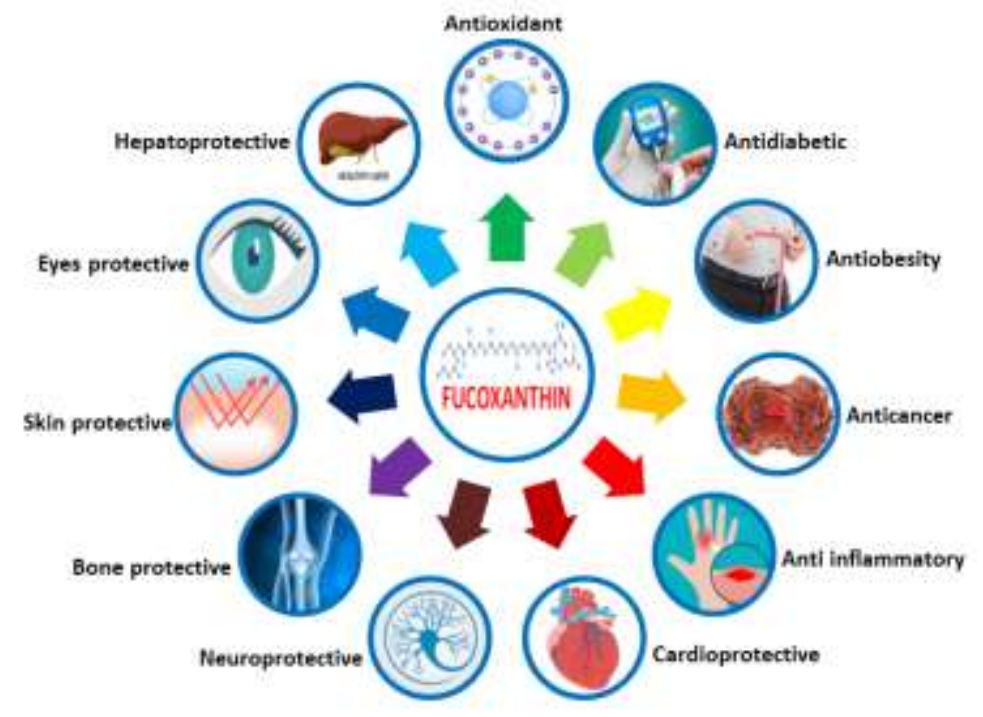

Fig. 2. The biological activities of fucoxanthin for human health

\section{Dietary and Safety}

There are two derivatives of fucoxanthin in the metabolism of mammals, namely fucoxanthinol and amarouciaxanthin A. In the digestive tract of mammals, the dietary fucoxanthin is hydrolyzed into fucoxanthinol. Then, in the liver, fucoxanthinol is dehydrogenated to amarouciaxanthin A. [167] (Fig. 3). Maeda [168] and Zhang et al., [169] reported that the fucoxanthin in common toxicity assay is a safe component as a food of functional, and safe pharmaceutical ingredient. Based on in vivo assay of the animal, it has no adverse effects of fucoxanthin supplementation. Additionally, macro brown algae, such Undaria pinnativida (Wakame) that contain much fucoxanthin has been consumed as edible algae for centuries in Eastern Asian countries [168]. 


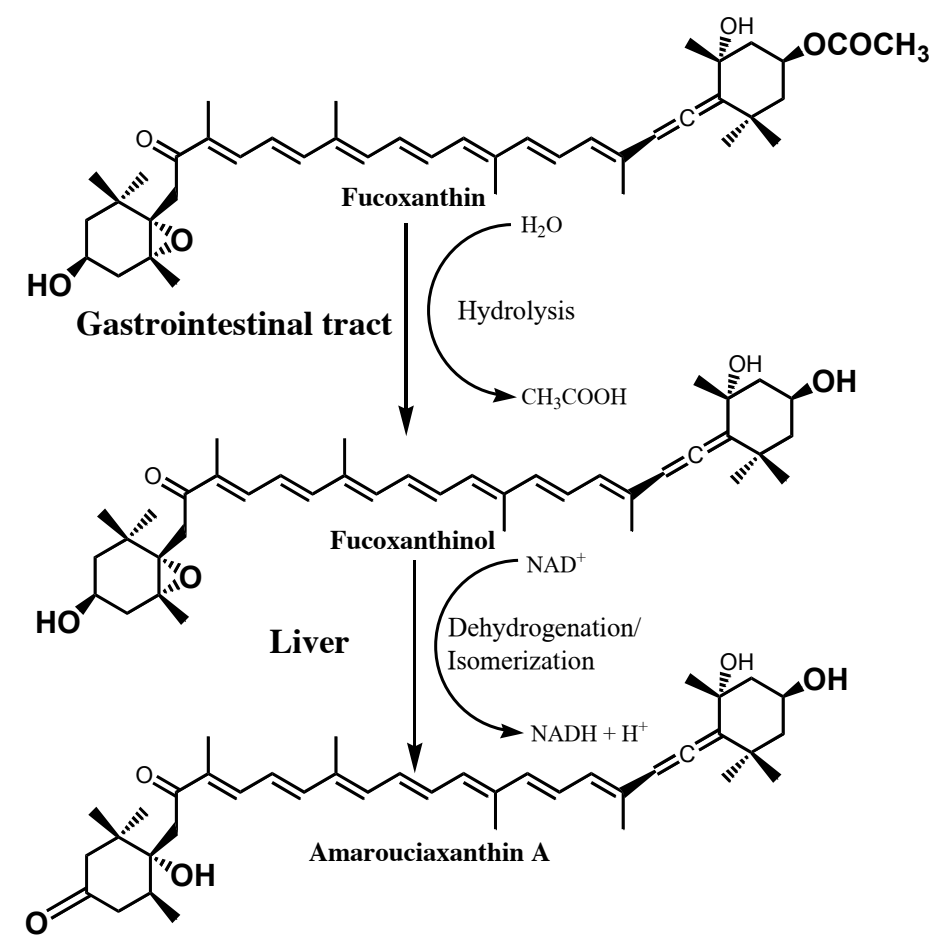

Fig. 3. Two derivatives of the fucoxanthin metabolism in mammals

\section{Potential Application of Fucoxanthin in Nutra- and Cosmeceutical Industry}

Fucoxanthin reported exhibit an array of beneficial biological activities on human health [169] including antidiabetic, antiobesity, antioxidant, anti-inflammatory, hepatoprotective, neuroprotective, osteoprotective, and eyes protective effect. Then, Muradian et al., [170] reported that fucoxanthin and its derivatives consumption as food supplements are good options for the treatment of metabolic syndrome and the prevention of obesity, diabetes type 2 , and disease of the heart.

Furthermore, fucoxanthin exerts strong anti-inflammatory and antioxidant effects. This compound can be applied as photoprotection of skin to inhibit the negative effects of UV radiation, or as an ingredient of Nutra- and cosmeceutical to prevent the diseases of oxidative stress [171,172]. Hence, fucoxanthin has great potential for application in Nutra[173], and cosmeceutical industries [174]. Galasso et al. [175] reported that there are main industrially produced fucoxanthin as a Nutra-, and cosmeceutical application, namely both of the industries were in China, such as Leili Natural Products Co., Ltd and AlgaNova International. In the future, fucoxanthin and its derivatives would be important for human health, contributing to the beauty industry, and playing an important in the prevention of cancer and the disease related to lifestyle. 


\section{Conclusion}

Fucoxanthin is found in macroalgae, especially brown algae, and microalgae, or a number of diatoms. The chemical structure of this xanthophyll is unique and that confers its biological activities. Then, based on their bioactivities, fucoxanthin has a wide range of potential applications and might be applied in both the Nutra- and cosmeceutical industries.

\section{References}

1. T. Maoka, J. Nat. Med 74 (2020)

2. $\quad$ N. Irvani, R. Hajiaghaee, A.R. Zarekarizi, J. Med. Plants 17, 6 (2018)

3. H.R.B. Raghavendran, A. Sathivel, S. Rekha, Gastric and hepatic protective effects of algal components (Woodhead Publishing Limited, 2013)

4. T. Rengarajan, R. Rajendran, N. Nandakumar, M.P. Balasubramanian, I. Nishigaki, Nutrients, 5, 4978 (2013)

5. T.M. Karpiński, A. Adamczak, Antioxidants 8, 239 (2019)

6. S. Wang, S.K. Verma, I.H. Said, L. Thomsen, M.S. Ullrich, N, Kuhnert, Microb. Cell Fact 17, 110 (2018)

7. S. Xia, K. Wang, L. Wan, A. Li, Q. Hu, C. Zhang, Mar. Drugs 11, 2667 (2013)

8. N. Abu-Ghannam, E. Shannon, Seaweed Carotenoid, Fucoxanthin, as Functional Food, in Microbial Functional Foods and Nutraceuticals, (John Wiley \& Sons, UK, 2017)

9. R. Aisyah, R. Rachmat, D. Rahmat, D. Noviendri, J. Ilmiah Kes 19, 59 (2020)

10. K. Teramukai, S. Kakui, F. Beppu, M. Hosokawa, K. Miyashita, Innov. Food Sci. Emerg. Technol 60, 102302 (2020)

11. C. Osório, S. Machado, J. Peixoto, S. Bessada, F.B. Pimentel, R.C. Alves, M.B.P.P. Oliveira, Separations 7, 33 (2020)

12. E.J. Jang, S.C. Kim, J-H. Lee, J.R. Lee, I.K. Kim, S.Y. Baek, Y.W. Kim, BMC Complement. Altern. Med 18, 97 (2018)

13. J. Peng, J-P. Yuan, C-F. Wu, J-H. Wang, Mar. Drugs 9, 1806 (2011)

14. M. Tanabe, Y. Ueno, M. Yokono, J-R. Shen, R. Nagao, S. Akimoto, Photosynth. Res (2020)

15. R. Nagao, Y. Ueno, S. Akimoto, J-R. Shen, Photosynth. Res (2020)

16. J. Matos, C. Cardoso, A. Gomes, A.M. Campos, P. Fale, C. Afonso, N.M. Bandarra, Food Funct 10, 7333 (2019)

17. E. Susanto, A.S. Fahmi, M. Hosokawa M, K. Miyashita, Mar. Drugs 17, 630 (2019)

18. L-J. Wang, Y. Fan, R.L. Parsons, G-R. Hu, P-Y. Zhang, F-L. Li, Mar. Drugs 16, 33 (2018b)

19. Z. Yi, M. Xu, M. Magnusdottir, Y. Zhang, S. Brynjolfsson, W. Fu, Mar. Drugs 13, $6138(2015)$

20. B. Guo, B. Liu, B. Yang, P. Sun, X. Lu, J. Lu, F. Chen, Mar. Drugs 14, 125 (2016)

21. E. Shannon E, N, Abu-Ghannam N, Int. J. Food Sci. Technol (2018).

22. M.K. Yousefi, M.S. Hashtroudi, A.M. Moradi, A.R.Ghasempur, Global J. Environ. Sci. Manage 4, 81(2018)

23. S. Xu, W.C. Liao, W. Chen, B. Kang, J. Chen, Y. Lin, IOP Conference, Series: Earth and Environmental Science 146, 012077 (2018)

24. Y-C. Chen, C-Y. Cheng, C-T. Liu, Y-M. Sue, T-H. Chen, Y-H. Hsu, P-A. Hwang, CH. Chen, J. Ethnopharmacol 224, 391 (2018)

25. T.H.P. Brotosudarmo, Heriyanto, Y. Shioi, Indriatmoko, M.A.S. Adhiwibawa, R. Indrawati, L. Limantara, Philipp. J. Sci 147, 47 (2018) 
26. H. Ravi, N. Kurrey, Y. Manabe, T. Sugawara, V. Baskaran, Mater. Sci. Eng. C 91, $785(2018)$

27. A.T. Getachew, P.S. Saravana, Y.J. Cho, H.C. Woo, B.S. Chun, J. $\mathrm{CO}_{2}$ Util 25, 137 (2018)

28. D.T. Vo, P.S. Saravana, H-C. Woo, B-S. Chun, J. $\mathrm{CO}_{2}$ Util 26, 359 (2018)

29. M.W.S. Lim, K.M. Tan, L.Y. Chew, K.W. Kong, S.W. Yan, J. Aquat. Food Prod. Technol (2018)

30. H. Maeda, S. Fukuda, H. Izumi, N. Saga, Mar. Drugs 16, 0255 (2018)

31. Z.A. Deviyani, K. Basah, A. Bahtiar, Int. J. Morphol 36, 979 (2018)

32. X. Sun, Y. Xu, L. Zhao, H. Yan, S. Wang, D. Wang, RSC Adv 8, 35139 (2018)

33. N. Zaharudin, D. Staerk, L.O. Dragsted, Food Chem (2018)

34. H. Li, Y. Xu, X. Sun, S. Wang, J. Wang, J. Zhu, D. Wang, L. Zhao, Food Hydrocol (2018)

35. S. Komba, E. Kotake-Nara, W. Tsuzuki, Mar. Drugs 16, 275 (2018)

36. S.C. Foo, F.M. Yusoff, M.Y. Imam MU, J.B. Foo, N. Ismail, N.H. Azmi, Y.S. Tor, N.M.H. Khong, M. Ismail, Biotechnol. Rep 20, (2018)

37. H. Wang, Y. Zhang, L. Chen L, W. Cheng, T. Liu, Bioprocess Biosyst. Eng 41, 1061 (2018a)

38. G.D. Lena, I. Casini, M. Lucarini, G. Lombardi-Boccia, Food Res. Int (2018)

39. M.S. Sahin, M.I. Khazi, Z. Demirel, M.C. Dalay, Biocatal. Agric. Biotechnol (2018)

40. X. Lu, H. Sun, W. Zhao, K-W. Cheng, F. Chen, B. Liu, Mar. Drugs 16, 219 (2018)

41. P. Sun, C-C. Wong, Y. Li, Y. He, X. Mao, T. Wu, Y. Ren, F. Chen, Food Chem (2018)

42. S. Xia, B. Gao, J. Fu, J. Xiong, C. Zhang, J. Biosci. Bioeng 1 (2018)

43. D.D McClure, A. Luiz, B. Gerber, G.W. Barton, J.M. Kavanagh, Algal Res 29, 41 (2018)

44. W. Zhang, F. Wang, B. Gao, L. Huang, C. Zhang, Algal Res 32, 193 (2018)

45. H. Staleva-Musto, R. West, M. Trathnigg, D. Bína, R. Latvín, T. Polívka, Faraday Discuss (2018)

46. Z. Yi, Y. Su, M. Xu, A. Bergmann, S. Ingthorsson, O. Rolfsson, K. Salehi-Ashtiani, S. Brynjolfsson, W. Fu, Mar. Drugs 16 (2018)

47. A. Delbrut, P. Albina, T. Lapierre, R. Pradelles, E. Dubreucq, Molecules (2018)

48. A. Gille, U. Neumann, S. Louis, S.C. Bischoff, K. Briviba, J. Funct. Foods 49, 285 (2018)

49. I-K. Mok, J.K. Lee, J.H. Kim, C-H. Pan, S.M. Kim, Food Chem (2018)

50. M.M.A. Nur, W. Muizelaar, P. Boelen, A.G.J. Buma, J. Appl. Phycol (2018)

51. E. Bigagli, L. Cinci, A. Niccolai, N. Biondi, L. Rodolfi, M. D’Ottavio, M. D’Ambrosio, M. Lodovici, M.R. Tredici, C. Luceri, Algal Res 34, 244 (2018)

52. U. Kim, D-H. Cho, J. Heo, H-S. Kim, J. Mar. Biosci. Biotechnol_11, 14 (2019)

53. T. Ishika, D.W. Laird, P.A. Bahri, N.R. Moheimani, J. Appl. Phycol 31, 1535 (2019)

54. J. Su, K. Guo, M. Huang, Y. Liu, J. Zhang, L. Sun, D. Li, K-L. Pang, G. Wang, L. Chen, Z. Liu, Y. Chen, Q. Chen, L. Huang, Front. Pharmacol 10, 906 (2019)

55. M. Peraman, S. Nachimuthu, Phcog. Mag 15, S243 (2019)

56. E. Medina, P. Cerezal, J. Morales, M.C. Ruiz-Domínguez, DYNA 86, 174 (2019)

57. H-L. Wu, X-Y. Fu, W-Q. Cao, W-Z. Xiang, Y-J. Hou, J-K. Ma, Y. Wang, C-D. Fan, J. Agric. Food Chem 67, 2212 (2019)

58. B. Guo, Yang, X. Pang, T. Chen, F. Chen, K-W. Cheng, Food Funct (2019)

59. B. Guo, T. Oliviero, V. Fogliano, V. Fogliano, Y. Ma, F. Chen, E. Capuano, J. Agric. Food Chem (2019)

60. X. Lu, B. Liu, Y. He, B. Guo, H. Sun, F. Chen, Biores. Technol 294, 122145 (2019)

61. Y. Cui, S.R. Thomas-Hall, P.M. Schenk, Food Chem 297, 124937 (2019a) 
62. H. Cui, H. Ma, Y. Cui, X. Zhu, S. Qin, R. Li, J. Biosci. Bioeng (2019b)

63. X. Yuan, L. Liang, K. Liu, L. Xie, L. Huang, W. He, Y. Chen, T. Xue, J.Appl. Phycol (2019)

64. A. Gille,B. Stojnic, F. Derwenskus, A. Trautmann, U. Schmid-Staiger, C. Posten, K. Briviba, A. Palou, M.L. Bonet, J. Ribot, Nutrients 11, 796 (2019)

65. U. Neumann, F. Derwenskus, V.F. Flister, U. Schmid-Staiger, T. Hirth, S.C. Bischof, Antioxidants 8,183 (2019)

66. R. Nagao, Y. Ueno, M. Yokono, J-R. Shen, S. Akimoto, Photosynth. Res (2019)

67. A. Kawee-ai, A.T. Kim, S.M. Kim, J. Oceanol. Limnol 37, 928 (2019)

68. S.Y. Koo, J-H. Hwang, S-H. Yang, J-I. Um, K.W. Hong, K. Kang, C-H. Pan, K.T. Hwang, S.M. Kim, Mar. Drugs 17, 311 (2019)

69. R.U. Arifah, S. Sedjati, E. Supriyantini, A. Ridlo, Bul. Oseano. Marina 8, 25 (2019)

70. S. Mohamadnia, O. Tavakoli, M.A. Faramarzi, Z. Shamsollahi, Aquaculture (2019)

71. R. Frassini, Y.P. da Silva, S. Moura, L.Z. Villela, A.P. Martins, P. Colepicolo, M.T. Fujii, N.S. Yokoya, C.M.P. de Pereira, V.R.Z.B. Pereira, J.A.P. Henriques, M. Roesch-Ely, Adv. Biol. Chem 9, 167 (2019)

72. E. Susanto, A.S. Fahmi, M. Hosokawa, K. Miyashita, Mar. Drugs 17, 630 (2019)

73. P. Paudel, S.H. Seong, H.A. Jung, J.S. Choi, Chem-Biol. Interact 310, 108757 (2019)

74. Y-T. Chau, H-Y. Chen, P-H. Lin, S-M. Hsia, Mar. Drugs 17 (2019)

75. Z-L. Kong, S. Sudirman, Y-C. Hsu, C-Y. Su, H-P. Kuo, Int. J. Mol. Sci 20, 4485 (2019)

76. P.S. Saravana, K. Shanmugapriya, C.R.N. Gereniu, S-J Chae, H.W. kang, H-C. Woo, B-S. Chun, Ultrason - Sonochem 55, 105 (2019)

77. M. Terasaki, Y. Kuramitsu, M. Kojoma, S-Y. Kim, T. Tanaka, H. Maeda, K. Miyahita, C. Kawagoe, S. Kohno, M. Mutoh, J. Funct. Foods (2019).

78. J. Wang, Y. Ma, J. Yang, L. Jin, Z. Gao, L. Xue, L. Hou, L. Sui, J. Liu, X. Zou, J. Cell. Mol. Med 1 (2019)

79. S.Yin, M. Shibata, T. Hagiwara, Food Sci. Technol. Res 25, 765 (2019)

80. S.C. Foo, N.M.H. Khong, F.M. Yusoff, Algal Res 51, 102061 (2020)

81. S. Akimoto, Y. Ueno, M. Yokono, J-R. Shen, R. Nagao, Photosynth. Res (2020)

82. C.A. Popovich, M.B. Faraoni, A. Sequeira, Y. Daglio, L.A. Martin, A.M. Martínez, M.C. Damiani, M.C. Matulewicz, P.I. Leonardi, Algal Res 51,102030 (2020)

83. S. Gérin, T. Delhez, A. Corato, C. Remacle, F. Franck, J. Appl. Phycol 32, 1581 (2020)

84. R. Yang, D. Wei, Front. Bioeng. Biotechnol 8, 820 (2020)

85. R. Nagao, M. Yokono, Y. Ueno, J-R. Shen, S. Akimoto, J. Phys. Chem. B (2020)

86. S.Y. Kang, H. Kang, J.E. Lee, C.S. Jo, C.B. Moon, J. Ha, J.S. Hwang, J. Choi, J. Cosmet. Sci 71, 53 (2020)

87. T-B. Hao, Y-F. Yang, S. Balamurugan, D-W. Li, W-D. Yang, H-Y. Li, Algal Res 47,101872 (2020)

88. T.K. Marella, A. Tiwari, Biores. Technol 307, 123245 (2020)

89. R.G. de Oliveira-Júniora, R. Grougnet, P-E. Bodet, A. Bonnet, E. Nicolau, A. Jebali, J. Rumin, L. Picot, Algal Res 51, 102035 (2020)

90. F. Gao, I. Teles, R.H. Wijffels, M.J. Barbosa, Biores. Technol (2020)

91. R. Gallego, C. Tardif, C. Parreira, T. Guerra, M.J. Alves, E. Ibáñez, M. Herrero, J. Sep. Sci 43,1967 (2020)

92. R.S.N. Tavares, C.M. Kawakami, K.d.C. Pereira, G.T.d. Amaral, C.G. Benevenuto, S.S. Maria-Engler, P. Colepicolo, H.M. Debonsi, L.R. Gaspar, Antioxidants 9, 328 (2020)

93. N. Oliyaei, M. Moosavi-Nasab, A.M. Tamaddon, M. Fazeli, Food Sci. Nutr 8, 226 (2020) 
94. P-T. Wang, S. Sudirman, M-C. Hsieh, J-Y. Hu, Z-L. Kong, Biomed.Pharmacother 125, $109992(2020)$

95. K. Teramukai, S. Kakui, F. Beppu, M. Hosokawa, K. Miyashita, Innov. Food Sci. Emerg. Technol 60, 102302 (2020)

96. N.K.E. Wati, L. Suhendra, N.M. Wartini, J. Reka. Manaj. Agroindustr 8, 80 (2020)

97. V. Raji, C. Loganathan, G. Sadhasivam, S. Kandasamy, K. Poomani, P. Thayumanavan, Int. J. Biol. Macromol 148, 696 (2020)

98. K. Thiyagarasaiyar, B-H. Goh, Y-J. Jeon, Y-Y. Yow, Mar. Drugs 18 (2020)

99. J. Fabrowska, B. Leska, G. Schroeder, B. Messyasz, M. Pikosz, Biomass and extracts of algae as materials for cosmetics. In Marine Algae Extracts, Processes, Products, and Applications, (Wiley-VCH-Verl: Weinheim, Germany, 2015)

100. N. Mezzomo, S.R.S. Ferreirra, J. Chem 2016 (2016)

101. E. Reverchon, I.D. Marco, J. Supercrit. Fluids 38, 146 (2006)

102. L.M. Kopas, J.J. Warthesen, J. Food Sci 60, 773 (1995)

103. P.T.V. Rosa, M.M.A. Meireles, J. Supercrit. Fluids 34, 109 (2005)

104. E.M.Z. Michielin, L.F.V. Bresciani, L. Danielski, R.A. Yunes, S.R.S. Ferreira, J. Supercrit. Fluids 33, 131 (2005)

105. B. Díaz-Reinoso, A. Moure, H. Domínguez, J.C. Parajó, J. Agric. Food Chem 54, $2441(2006)$

106. C. Turner, J.W. King, L. Mathiasson, J. Chromatogr. A 936, 215 (2001)

107. T. Rengarajan, R. Rajendran, N. Nandakumar, M.P. Balasubramanian, I. Nishigaki, Nutrients 5, 4978 (2013)

108. N. Takatani, Y. Kono, F. Beppu, Y. Okamatsu-Ogura, Y. yamano, K. Miyashita, M. Hosokawa, Biochem. Biophys. Res. Commun (2020)

109. S. Li, X. Ren X, Y. Wang, J. Hu, H. Wu, S. Song, C. Yan, Food Funct (2020)

110. M. Liu, W. Li, Y. Chen, X. wan, J. Wang, Life Sci 255, 117850 (2020)

111. C. Natsume, N. Aoki, T. Aoyama, K. Senda, M. Matsui, A. Ikegami, K. Tanaka, Y-T. Azuma, T. Fujita, Int. J. Mol. Sci 21, 2180 (2020)

112. J. Zheng, X. Tian, W. Zhang, P. Zheng, P. Huang, G. Ding, Z. Yang, Mar. Drugs 17, 552 (2019)

113. A. Rodríguez-Luna, J. Ávila-Román, H. Oliveira, H. Oliveira, V. Motilva, E. Talero, Mar. Drugs 17, 451 (2019)

114. Y-P. Yang, Q-Y. Tong, S-H. Zheng, M-D. Zhou, Y-M. Zheng, T-T. Zhou, Nat. Prod. Res 34 (2018)

115. J-H. Choi, N-H. Kim, S-J. Kim, H-J. Lee, S. Kim, J. Biochem. Mol. Toxicol 30, 111 (2016)

116. X. Jin, T.T. Zhao, D. Shi, M.B. Ye, Q. Ye, Drug Dev. Res 80, 209 (2019)

117. C.L. Liu, A.L. Liang, M.L. Hu, Toxicol. in Vitro 25, 1314 (2011)

118. M-N. Woo, S-M. Jeon, H-J. Kim, M-K. Lee, S-K. Shin, Y.C. Shin, Y-B. Park, M-S. Choi, Chem-Biol. Interact 186, 316 (2010)

119. F. Wang, H. Zhang, G. Lv, Z. Liu, X. Zheng, X. Wu, Phcog. Mag 16, 214 (2020)

120. H. Yoshida, H. Yanai, K. Ito, Y. Tomono, T. Koikeda, H. Tsukahara, N. Tada, Atherosclerosis 209, 520 (2010)

121. M. Matsumoto, M. Hosokawa, N. Matsukawa, M. Hagio, A. Shinoki, M. Nishimukai, K. Miyashita, T. Yajima, H. Hara, Eur. J. Nutr 49, 243 (2010)

122. M. Terasaki, M. Ikuta, H. Kojima, T. Tanaka, H. Maeda, K. Miyashita, M. Mutoh, J. Clin. Med 9, 90 (2020)

123. Y. Li, L. Tao, L. Bao, A. Chinnathambi, S.A. Alharbi, J. Cui, Phcog. Mag 16, 311 (2020)

124. Y. Long, X. Cao, R. Zhao, S. Gong, L. Jin, C. Feng, Environ. Toxicol 1 (2020) 
125. R.K. Saini, Y-S. Keum, M. Daglia, K.R. Rengasamy, Pharmacol. Res 157, 104830 (2020)

126. I. Jaswir, D. Noviendri, M. Taher, F. Mohamed, F. Octavianti, W. Lestari, A.G. Mukti, S. Nirwandar, B.B.H. Almansori, Molecules 24, 947 (2019)

127. M. Terasaki, N. Matsumoto, R. Hashimoto, T. Endo, H. Maeda, J. Hamada, K. Osada, K. Miyashita, M. Mutoh, J. Clin. Biochem. Nutr 64, 52 (2019)

128. Z. Wang, H. Li, M. Dong, P. Zhu, Y. Cai, J. Cancer Res. Clin. Oncol 145, 293 (2019)

129. H-L. Wu, X-Y. Fu, W-Q. Cao, W-Z. Xiang, Y-J. Hou, J-K. Ma, Y. Wang, C-D. Fan, J. Agric. Food Chem 67, 2212 (2019)

130. Y. Jin, S. Qiu, N. Shao, J. Zheng, Med. Sci. Monit 24, 11 (2018)

131. M. Sujatha, P. Suganya, V. Pradeepa, Int. J. Innov. Res. Sci. Eng. Technol 6, 16734 (2017)

132. K. Miyashita, F. Beppu, M. Hosokawa M, X. Liu, S. Wang, Arch. Biochem. Biophys 686, $108364(2020)$

133. H.A. Jung, M.N. Islam, C.M. Lee, H.O. Jeong, H.Y. Chung, H.C. Woo, J.S. Choi, Fish. Sci 78, 1321 (2012)

134. S. Nishikawa, M. Hosokawa, K. Miyashita, Phytomedicine 19, 389 (2012)

135. X. Sun, H. Zhao, Z. Liu, X. Sun, D. Zhang, S. Wang, Y. Xu, G. Zhang, D. Wang, J. Agric. Food Chem 68, 5118 (2020)

136. S. Zarei, H. Hosseinian, J. Mar. Med 1, 129 (2019)

137. A. Grasa-López, Á. Miliar-García, L. Quevedo-Corona, N. Paniagua-Castro, G. Escalona-Cardoso, E. Reyes-Maldonado, M-E. Jaramillo-Flores, Mar. Drugs 14 (2016)

138. M-J. Seo, Y-J. Seo, C-H. Pan, O-H. Lee, K-J. Kim, B-Y. Lee, Phytother. Res, 30, $1802(2016)$

139. M.A. Gammone, N. D’Orazio, Mar. Drugs 13, 2196 (2015)

140. Z. Guvatova, A. Dalina, E. Marusich, E. Pudova, A. Snezhkina, G. Krasnov, A. Kudryavtseva, S. Leonov, A. Moskalev, Mech. Ageing Dev (2020)

141. G. Yang, L. Jin, D. Zheng, X. Tang, J. Yang, L. Fan, X. Xie, Mar. Drugs 17, 702 (2019)

142. T.T. Dang, M.C. Bowyer, I.A.V. Altena, C.J. Scarlett, Int. J. Food Sci. Technol 53, 174 (2018)

143. Y. Zhang, H. Fang, Q. Xie, J. Sun, R. Liu, Z. Hong, R. Yi, H. Wu, Molecules 19, $2100(2014)$

144. N. Molina, A.C. Morandi, A.P. Bolin, R. Otton, Int. Immunopharmacol 22, 41 (2014)

145. A. Kawee-ai, A. Kuntiya, S.M. Kim, Nat. Prod. Commun, 8, 1381 (2013)

146. A.W. Ha, S.J. Na, W.K. Kim, Nutr. Res. Pract 7, 475 (2013)

147. Y-J. Lee, G-W. Nam, Cosmetics 7, 14 (2020)

148. S.Y. Kang, H. Kang, J.E. Lee, C.S. Jo, C.B. Moon, J. Ha, J.S. Hwang, J. Choi, J. Cosmet. Sci 71, 53 (2020)

149. R.S.N. Tavares, S.S. Maria-Engler, P. Colepicolo, H.M. Debonsi, M. Schäfer-Korting, U. Marx, L.R, Gaspar, C. Zoschke, Pharmaceutics 12, 136 (2020b)

150. L.M. Cordenonsi, A. Santer, R.M. Sponchiado, N.R. Wingert, R.P. Raffin, E.E.S. Schapoval, AAPS PharmSciTech 21, 32 (2020)

151. L.M. Cordenonsi, A. Faccendini, M. Catanzaro, M. Catanzaro, M.C. Bonferoni, S. Rossi, L. Malavasi, R.P. Raffin, E.E.S. Schapoval, C. Lanni, G. Sandri, F. Ferrari, Int. J. Pharm 567, 118487 (2019)

152. A. Rodríguez-Luna, J. Ávila-Román, M.L. González-Rodríguez, M.J. Cózar, A.M. Rabasco, V. Motilva, E. Talero, Mar. Drugs 16, 378 (2018) 
153. M. Matsui, K. Tanaka, N. Higashiguchi, H. Okawa, Y. Yamada, K. Tanaka, S. Taira, T. Aoyama, M. Takanishi, C. Natsume, Y. Takakura, N. Fujita, T. Hashimoto, T. Fujita, J. Pharmacol. Sci 132, 55 (2016)

154. X-S. Zhang, Y. Lu, T. Tao, H. Wang, G-J. Liu, C. Liu, D-Y. Xia, C-H. Hang, W. Li, Mol. Neurobiol (2020)

155. G. Sun, T. Xin, R. Zhang, C. Liu, Q. Pang, Phcog. Mag 16, 51 (2020)

156. M. Alghazwi, S. Smid, I. Musgrave, W. Zhang, Neurochem. Int 124, 215 (2019)

157. L. Hu, W. Chen, F. Tian, C. Yuan, H. Wang, H. Yue, Biomed. Pharmacother 106, $1484(2018)$

158. J. Yu, J-J. Lin, R. Yu, S. He, Q-W. Wang, W. Cui, J-R. Zhang, Food Nutr. Res 61, 1304678 (2017)

159. D. Zhao, S-H. Kwon, Y.S. Chun, M-Y. Gu, H.O. Yang, Neurochem. Res 42, 667 (2017)

160. J. Lin, J. Yu, J. Zhao, K. Zhang, J. Zheng, J. Wang, C. Huang, J. Zhang, X. Yan, W.H. Gerwick, Q. wang, W. Cui, S. He, Oxid. Med. Cell. Longev 2017, 6792543 (2017b)

161. L. Zhang, H. Wang, Y. Fan, Y. Gao, X. Li, Z. Hu, K. Ding, Y. Wang, X. Wang, Scient. Rep 7, 46763 (2017)

162. L. Guo, M. Dang, Q. Song, W. Zhang, B. Li, Phcog. Mag, 16, 242 (2020)

163. S.K. Das, R. Ren, T. Hashimoto, K. Kanazawa, J. Agric. Food Chem 58, 6090 (2010)

164. S-J. Chen, C-J. Lee, T-B. Lin, H-Y. Peng, H-J. Liu, Y-S. Chen, K-W. Tseng, Mar. Drugs 17, 152 (2019)

165. S-J. Chen, C-J. Lee, T-B. Lin, H-J. Liu, S-Y. Huang, J-Z. Chen, K-W. Tseng, Mar. Drugs 14, 13 (2016)

166. Y. Liu, L. Meng, X. Zhang, Q. Chen, C. Haixiu, L. Sun, G-M. Liu. J. Agric. Food Chem (2016).

167. A. Asai, L. Yonekura, A. Nagao, British J. Nutr 100, 273 (2008)

168. H. Maeda, Anti-obesity and anti-diabetic activities of algae. Ch. 13 (Woodhead Publishing Limited, 2013)

169. H. Wu, T. Li, G. Wang, S. Dai, H. He, W. Xiang, Chin. J. Oceanol. Limnol 34, 391 (2016)

170. K. Muradian, A. Vaiserman, K-J. Min, V.E. Fraifeld, Nutr. Metabol. Cardiovasc. Dis 25, $891(2015)$

171. J.A. Nichols, S.K. Katiyar, Arch. Dermatol. Res 302, 71 (2010)

172. J-Y. Berthon, R. Nachat-Kappes, M. Bey, J-P. Cadoret, I. Renimel, E. Filaire, Free Radic. Res 51, 555 (2017)

173. M.F. Hossain, M. Rashid, T. Burniston, M. Ahmed, W. Wu, K.A. Kataye, R. Sidhu, M. Justice, S. Abdelfattah, J. Obes. Weight-Loss Medic 5, 031 (2019)

174. S-J. Heo, Y-J. Jeon, J. Photochem. Photobiol. B: Biol 95, 101 (2009)

175. C. Galasso, C. Corinaldesi, C. Sansone, Antioxidants 6 (2017) 